\title{
Optimal location of resources and Steiner symmetry in a population dynamics model in heterogeneous environments
}

\author{
Claudia Anedda and Fabrizio Cuccu
}

\begin{abstract}
The subject of this paper is inspired by Cantrell and Cosner (1989) and Cosner, Cuccu and Porru (2013). Cantrell and Cosner (1989) investigate the dynamics of a population in heterogeneous environments by means of diffusive logistic equations. An important part of their study consists in finding sufficient conditions which guarantee the survival of the species. Mathematically, this task leads to the weighted eigenvalue problem $-\Delta u=\lambda m u$ in a bounded smooth domain $\Omega \subset \mathbb{R}^{N}, N \geq 1$, under homogeneous Dirichlet boundary conditions, where $\lambda \in \mathbb{R}$ and $m \in L^{\infty}(\Omega)$. The domain $\Omega$ represents the environment and $m(x)$, called the local growth rate, says where the favourable and unfavourable habitats are located. Then, Cantrell and Cosner (1989) consider a class of weights $m(x)$ corresponding to environments where the total sizes of favourable and unfavourable habitats are fixed, but their spatial arrangement is allowed to change; they determine the best choice among them for the population to survive.

In our work we consider a sort of refinement of the result above. We write the weight $m(x)$ as sum of two (or more) terms, i.e. $m(x)=f_{1}(x)+f_{2}(x)$, where $f_{1}(x)$ and $f_{2}(x)$ represent the spatial densities of the two resources which contribute to form the local growth rate $m(x)$. Then, we fix the total size of each resource allowing its spatial location to vary. As our first main result, we show that there exists an optimal choice of $f_{1}(x)$ and $f_{2}(x)$ and find the form of the optimizers. Our proof relies on some results in Cosner, Cuccu and Porru (2013) and on a new property (to our knowledge) about the classes of rearrangements of functions. Moreover, we show that if $\Omega$ is Steiner symmetric, then the best arrangement of the resources inherits the same kind of symmetry. (Actually, this is proved in the more general context of the classes of rearrangements of measurable functions.)
\end{abstract}

\section{Resurssien optimaalinen sijainti ja Steinerin symmetria populaatiodynamiikan mallissa epätasalaatuisissa ympäristöissä}

Tiivistelmä. Tämän tutkimuksen aihetta ovat inspiroineet Cantrellin ja Cosnerin (1989) sekä Cosnerin, Cuccun ja Porrun (2013) työt. Cantrell and Cosner (1989) tutkivat populaatiodynamiikkaa epätasalaatuisissa ympäristöissä diffuusiotyyppisten logististen yhtälöiden avulla. Tärkeä osa heidän tutkimustaan on lajin säilymisen takaavien riittävien ehtojen löytäminen. Matemaattisesti tämä tehtävä johtaa painotettuun ominaisarvo-ongelmaan $-\Delta u=\lambda m u$ homogeenisilla Dirichlet'n reunaehdoilla rajoitetussa sileässä alueessa $\Omega \subset \mathbb{R}^{N}, N \geq 1$, missä $\lambda \in \mathbb{R}$ ja $m \in L^{\infty}(\Omega)$. Alue $\Omega$ kuvaa ympäristöä ja ns. paikallinen kasvuvauhti $m(x)$ kertoo, missä suotuisat ja epäsuotuisat elinympäristöt sijaitsevat. Cantrell ja Cosner (1989) tarkastelevat sellaisia olosuhteita kuvaavia painoja $m(x)$, joissa suotuisien ja epäsuotuisien elinympäristöjen kokonaisalat ovat kiinteitä, mutta niiden sijainnin sallitaan vaihdella; sitten he määrittävät niiden parhaan sijainnin populaation selviytymisen kannalta.

Tässä työssä tarkastelemme em. tulosten eräänlaista tarkennusta. Kirjoitamme painon $m(x)$

https://doi.org/10.54330/afm.113547

2020 Mathematics Subject Classification: Primary 47A75, 35J25, 35Q80.

Key words: Population dynamics, eigenvalue problem, indefinite weight, optimization, Steiner symmetry.

(c) 2022 The Finnish Mathematical Society 
kahden (tai useamman) termin summana $m(x)=f_{1}(x)+f_{2}(x)$, missä $f_{1}(x)$ ja $f_{2}(x)$ kuvaavat kahden sellaisen resurssin sijaintia, jotka yhdessä muodostavat paikallisen kasvuvauhdin $m(x)$. Kiinnitämme kummankin resurssin kokonaismäärän, mutta sallimme sen sijainnin vaihdella. Ensimmäisenä päätuloksenamme todistamme resurssien $f_{1}(x)$ ja $f_{2}(x)$ optimaalisen valinnan olemassaolon sekä etsimme sen muodon. Todistuksemme perustuu eräisiin Cosnerin, Cuccun ja Porrun (2013) tuloksiin sekä funktioiden uudelleenjärjestelyluokkia koskevaan (tietääksemme) uuteen ominaisuuteen. Lisäksi osoitamme, että jos $\Omega$ on Steinerin mielessä symmetrinen, niin resurssien paras järjestely perii saman symmetrian. (Itse asiassa todistamme tämän yleisemmin mitallisten funktioiden uudelleenjärjestelyluokille.)

\section{Introduction and main results}

In this paper we consider the weighted eigenvalue problem

$$
\begin{cases}-\Delta u=\lambda m(x) u & \text { in } \Omega, \\ u=0 & \text { on } \partial \Omega,\end{cases}
$$

where $\Omega \subset \mathbb{R}^{N}, N \geq 1$, is a bounded smooth domain with boundary $\partial \Omega$, the weight $m(x)$ belongs to $L^{\infty}(\Omega)$ and $\lambda \in \mathbb{R}$. When the set $\{x \in \Omega: m(x)>0\}$ (respectively, $\{x \in \Omega: m(x)<0\})$ has positive Lebesgue measure, problem (1) admits an increasing (decreasing) sequence of positive (negative) eigenvalues (see Section 2). Here, under the first assumption above, we are interested in the smallest positive eigenvalue $\lambda_{1}(m)$, which we will call the principal positive eigenvalue. More precisely, we study the minimization of $\lambda_{1}(m)$ when $m$ is chosen in an appropriate class of bounded measurable functions.

Problem (1) originates from the study of reaction-diffusion equations in mathematical ecology which dates to the pioneering work [21] of Skellam. Precisely, we deal with the following model examined by Cantrell and Cosner in [5] and [6]

$$
\begin{cases}v_{t}=d \Delta v+[m(x)-c v] v & \text { in } \Omega \times(0, \infty), \\ v(x, 0)=v_{0}(x) \geq 0 & \text { for } x \in \bar{\Omega}, \\ v(x, t)=0 & \text { on } \partial \Omega \times(0, \infty) .\end{cases}
$$

In $(2) v(x, t)$ represents the population density of a species inhabiting the region $\Omega$ in position $x$ at time $t$ surrounded by the hostile region $\mathbb{R}^{N} \backslash \Omega, v_{0}$ is the initial density and $c, d$ are positive constants describing the limiting effects of crowding and the diffusion rate of the population, respectively. The function $m(x)$ represents the local growth rate of the population, it is positive on favourable habitats and negative on unfavourable ones. Moreover, the homogeneous Dirichlet boundary conditions mean that the exterior of $\Omega$ is a deadly environment (any individual reaching the boundary dies). The aim of the papers [5] and [6] is to study how the spatial arrangements of favourable and unfavourable habitats in $\Omega$ affects the survival of the modelled population. The authors show that (2) predicts persistence for the population if $\lambda_{1}(m)<1 / d$. As a consequence, determining the best spatial arrangement of favourable and unfavourable habitats for the survival, within a fixed class of environmental configurations, results in minimizing $\lambda_{1}(m)$ over the corresponding class of weights. In [5] and [6] different aspects of model (2) have been extensively studied. This kind of problems has been investigated by many authors, mostly under Neumann boundary conditions (in this case the boundary $\partial \Omega$ acts as a fence and any individual reaching it returns to $\Omega$ ) or in the case of a periodic environment $\left(\Omega=\mathbb{R}^{N}\right.$ and $m(x)$ periodic). In particular, we mention Berestycki et al. [3] and Lou and 
Yanagida [17], who investigated how the fragmentation of the environment affects the persistence of the population, Roques and Hamel [19], who studied the optimal arrangement of resources by using numerical computation, Jha and Porru [15] who, among the other things, exhibited an example of symmetry breaking of the optimal arrangement of the local growth rate, Cosner et al. [9], who considered the minimization of $\lambda_{1}(m)$ in the framework of rearrangements of functions and Lamboley et al. [16], who investigated model (2) with Robin boundary conditions.

Our present study has two main aims, which we describe in details in what follows. Throughout the paper we consider the Lebesgue measure on $\mathbb{R}^{N}$ and denote by $|E|$ the measure of a measurable set $E$. Moreover, $\chi_{E}$ represents the usual characteristic or indicator function of the set $E$. In [5, Theorem 3.9] the authors consider the minimization of $\lambda_{1}(m)$ when $m$ is taken in the set $^{1}$

$$
\begin{gathered}
\mathcal{M}=\left\{m(x) \in L^{\infty}(\Omega):-m_{2} \leq m(x) \leq m_{1} \text { a.e. in } \Omega, \int_{\Omega} m d x=m_{3}\right. \\
\text { and } m(x)>0 \text { on a set of positive measure }\},
\end{gathered}
$$

where $m_{1}, m_{2}$ and $m_{3}$ are suitable fixed constants with $m_{1}$ and $m_{2}$ positive. The choice of the class $\mathcal{M}$ has the following biological meaning: the local growth rate of the population in each point of $\Omega$ has a value between the minimum $-m_{2}$ and the maximum $m_{1}$; moreover, the "total growth rate" is fixed and equal to $m_{3}$. This can be obtained by introducing different amounts of resources in the environment $\Omega$; here, in order to simplify our exposition, by the term "resource" we mean anything that affects the growth rate of the population both in positive (for example food) and in negative (for example predators) sense. How should we arrange the favourable and unfavourable habitats in $\Omega$ in order to maximize the chance of survival of the population? Theorem 3.9 in [5] establishes that an optimal choice consists in using only the maximum $m_{1}$ and the minimum $-m_{2}$ values of the local growth rate. In terms of the weight $m(x)$, this means that an optimal configuration is a distribution of "bang-bang" type, i.e. is a function which takes only two values in $\Omega$.

We note that in the previous approach the maximization of the chance of survival of the population is considered with constraints only on the local growth rate $m(x)$ and not on the single resource available in the environment $\Omega$. Indeed, the same local growth rate can be obtained with different blends and/or number of resources. For example, the same effect can be attained putting some amount of food in $\Omega$ or putting more food but introducing also some predators in the environment. In our work we decompose the local growth rate $m(x)$ into a sum of two terms $f_{1}(x)$ and $f_{2}(x)$ which we interpret as the spatial densities of different types of resources. Then, we fix the total amount of each resource and seek for their best location in $\Omega$ in order to maximize the chance of the population to survive. In mathematical terms, we consider the minimization of $\lambda_{1}(m)$ as the weight $m(x)$ varies in the class

$$
\begin{gathered}
\mathcal{M}=\left\{m(x) \in L^{\infty}(\Omega): m(x)=f_{1}(x)+f_{2}(x), f_{1} \in \mathcal{F}_{1}, f_{2} \in \mathcal{F}_{2},\right. \\
m(x)>0 \text { on a set of positive measure }\},
\end{gathered}
$$

where

$$
\mathcal{F}_{1}=\left\{f(x) \in L^{\infty}(\Omega):-p_{1} \leq f(x) \leq q_{1} \text { a.e. in } \Omega \text { and } \int_{\Omega} f d x=l_{1}\right\}
$$

\footnotetext{
${ }^{1}$ We follow the notation of [5, Theorem 3.9] except for the replacement of $m_{0}$ by $m_{3}, \bar{m}$ by $\check{m}$ and $\lambda_{1}^{+}$by $\lambda_{1}$.
} 
and

$$
\mathcal{F}_{2}=\left\{f(x) \in L^{\infty}(\Omega):-p_{2} \leq f(x) \leq q_{2} \text { a.e. in } \Omega \text { and } \int_{\Omega} f d x=l_{2}\right\},
$$

with $p_{1}, q_{1}, l_{1}$ and $p_{2}, q_{2}, l_{2}$ suitable fixed constants. Our first main result shows that a minimizer actually exists. As it can be expected, each component $f_{1}(x)$ and $f_{2}(x)$ of the optimal weight must be of "bang-bang" type. Moreover, we find that the two "optimal components" cannot be chosen independently. Indeed, they have a special feature: $f_{2}(x)$ has to be large (respectively, small) where $f_{1}(x)$ is large (small) as much as possible. To be precise, $f_{1}(x)$ and $f_{2}(x)$ must realize the equality sign in the Hardy-Littlewood inequality (6). We give a description of our minimizer in the following theorem.

Theorem 1. Let $\Omega \subset \mathbb{R}^{N}$ be a bounded smooth domain and $\mathcal{M}=\{m(x) \in$ $L^{\infty}(\Omega): m(x)=f_{1}(x)+f_{2}(x), f_{1} \in \mathcal{F}_{1}, f_{2} \in \mathcal{F}_{2}, m(x)>0$ on a set of positive measure $\}$, where $\mathcal{F}_{i}=\left\{f(x) \in L^{\infty}(\Omega):-p_{i} \leq f(x) \leq q_{i}\right.$ a.e. in $\Omega$ and $\left.\int_{\Omega} f d x=l_{i}\right\}$, with $p_{i}, q_{i}$ and $l_{i}$ constants such that $-p_{i}|\Omega|<l_{i}<q_{i}|\Omega|, i=1,2$, and $q_{1}+q_{2}>0$. Moreover, let $e_{i}=\left(p_{i}|\Omega|+l_{i}\right) /\left(p_{i}+q_{i}\right), i=1,2$. Then there exist two measurable sets $E, G \subseteq \Omega$, with $|E|=e_{1},|G|=e_{2}$, subject to the conditions

$$
\begin{cases}E \supset G & \text { if } e_{1}>e_{2} \\ E=G & \text { if } e_{1}=e_{2} \\ E \subset G & \text { if } e_{1}<e_{2}\end{cases}
$$

such that $\check{f}_{1}=q_{1} \chi_{E}-p_{1} \chi_{\Omega \backslash E} \in \mathcal{F}_{1}, \check{f}_{2}=q_{2} \chi_{G}-p_{2} \chi_{\Omega \backslash G} \in \mathcal{F}_{2}$ and $\check{m}=\check{f}_{1}+\check{f}_{2}(\in \mathcal{M})$ satisfies $\lambda_{1}(\check{m})=\inf \left\{\lambda_{1}(m): m \in \mathcal{M}\right\}$.

Note that $\check{m}$ takes at least two and at most three values in $\Omega$. The proof of Theorem 1 relies on some results contained in [9] and on a new result (to our knowledge) about the classes of rearrangements of functions (see Section 3). Incidentally, we note that, using the same theoretical machinery, Theorem 3.9 in [5] can easily be proved. Actually, the statement of Theorem 1 can be strengthen. Indeed, as a matter of fact, every minimizer of $\lambda_{1}(m)$ in the class $\mathcal{M}$ has the form described in Theorem 1. Unfortunately, this does not follow from [9] as it happens for Theorem 1; instead, it can be shown reasoning exactly as in the proof of Theorem 1.1 in [1]. Due to the length of this argument, we prefer to include this topic in a future paper.

A remarkable consequence of Theorem 1 is that, by a simple examination of $\check{f}_{1}+\check{f}_{2}$ (see also the proof of the theorem), problem $\inf _{m \in \mathcal{M}} \lambda_{1}(m)$ reduces to the following shape optimization problem (which can also be seen as a sort of optimal partition problem)

$$
\begin{aligned}
& \inf _{\substack{E, G \subseteq \Omega \\
|E|=e_{1},|G|=e_{2}}} \lambda_{1}\left(\left(q_{1}+q_{2}\right) \chi_{G}+\left(q_{1}-p_{2}\right) \chi_{E \backslash G}-\left(p_{1}+p_{2}\right) \chi_{\Omega \backslash E}\right) \quad \text { if } e_{1}>e_{2}, \\
& \quad \inf _{\substack{E \subseteq \Omega \\
|E|=e_{1}}} \lambda_{1}\left(\left(q_{1}+q_{2}\right) \chi_{E}-\left(p_{1}+p_{2}\right) \chi_{\Omega \backslash E}\right) \quad \text { if } \quad e_{1}=e_{2}
\end{aligned}
$$

and similarly when $e_{1}<e_{2}$. Furthermore, this problem has a solution.

We also note that Theorem 1 can be extended to the general case

$$
\begin{array}{r}
\mathcal{M}=\left\{m(x) \in L^{\infty}(\Omega): m(x)=\sum_{i=1}^{n} f_{i}(x), f_{i} \in \mathcal{F}_{i}\right. \\
\quad \text { and } m(x)>0 \text { on a set of positive measure }\},
\end{array}
$$


where

$$
\mathcal{F}_{i}=\left\{f(x) \in L^{\infty}(\Omega):-p_{i} \leq f(x) \leq q_{i} \text { a.e. in } \Omega \text { and } \int_{\Omega} f d x=l_{i}\right\},
$$

with $i=1, \ldots, n$. In this case, there exists a minimizer $\check{m}$ which takes at least two and at most $n+1$ values in $\Omega$.

The second main result of this paper concerns the case $\Omega$ is Steiner symmetric. In this situation, we prove the Steiner symmetry of all the minimizers $\check{m}$ of $\lambda_{1}(m)$, when $m$ varies in a class of rearrangements of a fixed bounded function. As byproduct we will obtain the Steiner symmetry of the minimizers in Theorem 1 and Theorem 3.9 in [5] (see Section 5). Two measurable functions $f, g: \Omega \rightarrow \mathbb{R}$ are said equimeasurable if the superlevel sets $\{x \in \Omega: f(x)>t\}$ and $\{x \in \Omega: g(x)>t\}$ have the same measure for all $t \in \mathbb{R}$. For a fixed $f \in L^{\infty}(\Omega)$ we call the set $\mathcal{G}(f)=\{g: \Omega \rightarrow$ $\mathbb{R}: g$ is measurable and $g$ and $f$ are equimeasurable $\}$ the class of rearrangements of $f$ (see Subsection 2.2). Roughly speaking, a set is Steiner symmetric if it is symmetric and convex relative to a hyperplane and a function is Steiner symmetric if any of its superlevel set is Steiner symmetric (see Section 5). $\mathbb{R}^{N-1}$.

In what follows we denote a point $x \in \mathbb{R}^{N}$ by $\left(x_{1}, x^{\prime}\right)$, where $x_{1} \in \mathbb{R}$ and $x^{\prime} \in$

Theorem 2. Let $\Omega \subset \mathbb{R}^{N}$ be a bounded smooth domain and assume it is Steiner symmetric with respect to the hyperplane $T=\left\{x=\left(x_{1}, x^{\prime}\right) \in \mathbb{R}^{N}: x_{1}=0\right\}$. Let $m_{0} \in L^{\infty}(\Omega)$ such that $\left\{x \in \Omega: m_{0}(x)>0\right\}$ has positive measure. Then every minimizer $\check{m}$ of the problem

$$
\inf \left\{\lambda_{1}(m): m \in \mathcal{G}\left(m_{0}\right)\right\}
$$

is Steiner symmetric relative to $T$.

An equivalent result is proved in [3]; however, here we propose a novel proof. The biological meaning of Theorem 2 is the following: if the region $\Omega$ is Steiner symmetric, for the population to survive the best environment is given when the favourable habitat is located far from the boundary $\partial \Omega$ and arranged in a Steiner symmetrical fashion. As a Steiner symmetric set is convex relative to a direction, the favourable habitat cannot be made, at least in that direction, of disconnected pieces. In other words, it should not be very fragmented. This conclusion is a well-known biological fact (see for example $[5,6,3,20]$ ).

Problem (1) with positive bounded weight $m(x)$ also has a well known physical interpretation: it models a vibrating membrane $\Omega$ with clamped boundary $\partial \Omega$ and mass density $m(x)$; the physical meaning of $\lambda_{1}(m)$ is the principal natural frequency of the membrane. Thus, the minimization of $\lambda_{1}(m)$ is physically equivalent to find the mass distribution of the membrane which gives the lowest principal natural frequency. Among many papers that consider this interpretation of problem (1), we recall [7, 10,11 , where the minimization of $\lambda_{1}(m)$ is addressed in the case of a class of weights which take only two positive values.

This paper is structured as follows. In Section 2 we describe the eigenvalue problem (1) in some details and summarize some known results about the minimization of $\lambda_{1}(m)$. Moreover, we recall the definition of class of rearrangements of a measurable function and some related properties we will need in the sequel. In Section 3 we show a new formula involving the classes of rearrangements, which we will use in Section 4 in order to prove Theorem 1. Finally, Section 5 contains the proof of Theorem 2. 


\section{Preliminaries}

We denote by $|E|$ the measure of an arbitrary Lebesgue measurable set $E \subseteq \mathbb{R}^{N}$ and, when $N=1$, we also write $|E|_{1}$. We identify two measurable sets $E, F$ that are equal up to a nullset, i.e. if $|E \backslash F \cup F \backslash E|=0$ and similarly for measurable functions equal almost everywhere.

2.1. The weighted eigenvalue problem. Let $\Omega \subset \mathbb{R}^{N}$ be a bounded smooth domain and $m \in L^{\infty}(\Omega)$. By $H_{0}^{1}(\Omega)$ and $W^{2,2}(\Omega)$ we denote the usual Sobolev spaces; we use the norm $\|u\|_{H_{0}^{1}(\Omega)}=\int_{\Omega}|\nabla u|^{2} d x$ (see [14]). Problem (1) is considered in weak form: $u \in H_{0}^{1}(\Omega)$ is a weak solution of (1) if

$$
\int_{\Omega} \nabla u \cdot \nabla \varphi d x=\lambda \int_{\Omega} m u \varphi d x \quad \forall \varphi \in C_{0}^{\infty}(\Omega) .
$$

A nontrivial solution of (1) is called an eigenfunction associated to the eigenvalue $\lambda$. It is easy to check that zero is not an eigenvalue of problem (1). The following proposition is a consequence of a result contained in [13].

Proposition 1. With the notation above we have:

i) if $|\{x \in \Omega: m(x)>0\}|>0$, then there is a divergent sequence $\left\{\lambda_{k}(m)\right\}_{k=1}^{\infty}$ of positive eigenvalues of problem (1);

ii) if $|\{x \in \Omega: m(x)<0\}|>0$, then there is a divergent sequence $\left\{\lambda_{-k}(m)\right\}_{k=1}^{\infty}$ of negative eigenvalues of problem (1).

The smallest positive eigenvalue $\lambda_{1}(m)$, which we call the principal positive eigenvalue, is simple and any associated eigenfunction is one-signed in $\Omega$ (see [13, Theorem 1.13]), and similarly for $\lambda_{-1}(m)$. In general, the eigenvalues of problem (1) form two monotone sequences

$$
0<\lambda_{1}(m)<\lambda_{2}(m) \leq \ldots \leq \lambda_{k}(m) \leq \ldots
$$

and

$$
\ldots \leq \lambda_{-k}(m) \leq \ldots \leq \lambda_{-2}(m)<\lambda_{-1}(m)<0,
$$

where every eigenvalue is repeated according to its multiplicity.

Moreover, by classical regularity results, any eigenfunction $u$ related to $\lambda_{1}(m)$ belongs to $H_{0}^{1}(\Omega) \cap W^{2,2}(\Omega) \cap C^{1, \beta}(\bar{\Omega})$ for every $\beta \in(0,1)$ (see [14]). The principal positive eigenvalue $\lambda_{1}(m)$ has a variational characterization also known as the Courant-Fischer Principle

$$
\frac{1}{\lambda_{1}(m)}=\max _{\substack{u \in H_{0}^{1}(\Omega) \\ u \neq 0}} \frac{\int_{\Omega} m u^{2} d x}{\int_{\Omega}|\nabla u|^{2} d x}
$$

Furthermore, each maximizer of (5) is an eigenfunction associated to $\lambda_{1}(m)$ (see Proposition 1.10 and the proof of Lemma 1.1 in [13]).

As described in Section 1, we consider the minimization of $\lambda_{1}(m)$ with respect to $m$, when $m$ belongs to a fixed class of bounded functions. Let us introduce in details the classes we are interested in.

2.2. Classes of rearrangements. Here we briefly recall some basic definitions and properties about the rearrangements of measurable functions. For a systematic and thorough treatment of this subject we refer the reader to [12]. 
Definition 1. Let $E \subset \mathbb{R}^{N}$ be a set of finite measure. Two measurable functions $f, g: E \rightarrow \mathbb{R}$ are called equimeasurable or rearrangements of one another if

$$
|\{x \in E: f(x)>t\}|=|\{x \in E: g(x)>t\}| \quad \forall t \in \mathbb{R} .
$$

Equimeasurability of $f$ and $g$ is denoted by $f \sim g$. Note that $\sim$ is an equivalence relation on the set of all measurable functions on $E$. Equimeasurable functions share global extrema and integrals as it is precisely stated in the following proposition.

Proposition 2. Let $E \subset \mathbb{R}^{N}$ be a set of finite measure and $f, g: E \rightarrow \mathbb{R}$ two measurable functions. If $f \sim g$, then

i) ess $\sup f=\operatorname{ess} \sup g$ and ess inf $f=\operatorname{ess} \inf g$;

ii) $f \in L^{1}(E)$ if and only if $g \in L^{1}(E)$, in which case $\int_{E} f d x=\int_{E} g d x$;

iii) if $F: \mathbb{R} \rightarrow \mathbb{R}$ is a Borel measurable function, then $F \circ f \sim F \circ g$.

For a proof see, for example, [12, Proposition 3.3].

Definition 2. Let $E \subset \mathbb{R}^{N}$ be a set of finite measure. For every measurable function $f: E \rightarrow \mathbb{R}$, the function $f^{*}:(0,|E|) \rightarrow \mathbb{R}$ defined by

$$
f^{*}(s)=\sup \{t \in \mathbb{R}:|\{x \in E: f(x)>t\}|>s\}
$$

is called the decreasing rearrangement of $f$.

It can be proved that $|\{x \in E: f(x)>t\}|=\left|\left\{s \in(0,|E|): f^{*}(s)>t\right\}\right|$ for all $t \in \mathbb{R}$ (see [12, Theorem 5.2]).

Proposition 3. Let $f$ be as in Definition 2. Then

i) $f^{*}$ is decreasing, right continuous and

$$
\lim _{s \rightarrow 0} f^{*}(s)=\operatorname{ess} \sup f \quad \text { and } \quad \lim _{s \rightarrow|E|} f^{*}(s)=\operatorname{essinf} f ;
$$

ii) $f \in L^{1}(E)$ if and only if $f^{*} \in L^{1}(0,|E|)$, in which case $\int_{E} f d x=\int_{0}^{|E|} f^{*} d s$;

iii) if $F: \mathbb{R} \rightarrow \mathbb{R}$ is a Borel measurable function, then $F \circ f \in L^{1}(E)$ if and only if $F \circ f^{*} \in L^{1}(0,|E|)$, in which case $\int_{E} F \circ f d x=\int_{0}^{|E|} F \circ f^{*} d s$;

iv) for any measurable function $g$ on $E$ we have $g \sim f$ if and only if $g^{*}=f^{*}$.

The proof easily follows from Definition 2 and Proposition 3.3 and 5.3 in [12].

Definition 3. Let $E \subset \mathbb{R}^{N}$ be a set of finite measure and $f, g \in L^{1}(E)$. We write $g \prec f$ if

$$
\int_{0}^{t} g^{*} d s \leq \int_{0}^{t} f^{*} d s \quad \forall 0 \leq t \leq|E| \quad \text { and } \quad \int_{0}^{|E|} g^{*} d s=\int_{0}^{|E|} f^{*} d s .
$$

Proposition 4. Let $E \subset \mathbb{R}^{N}$ be a set of finite measure and $f, g \in L^{1}(E)$. Then

i) $f \sim g$ if and only if $f \prec g$ and $g \prec f$;

ii) if $\alpha \leq f \leq \beta$ a.e. in $E$ with $\alpha, \beta \in \mathbb{R}$ and $g \prec f$, then $\alpha \leq g \leq \beta$ a.e. in $E$;

iii) the constant function $c=\frac{1}{|E|} \int_{E} f d x$ precedes $f$, i.e. $c \prec f$.

Property i) follows from Proposition 3 and Definition 3; the proof of ii) and iii) can be found in [12, Lemma 8.2].

Definition 4. Let $E \subset \mathbb{R}^{N}$ be a set of finite measure and $f: E \rightarrow \mathbb{R}$ a measurable function. We call the set

$$
\mathcal{G}(f)=\{g: E \rightarrow \mathbb{R}: g \text { is measurable and } g \sim f\}
$$

the class of rearrangements of $f$ or the set of rearrangements of $f$. 
Note that, for $1 \leq p \leq \infty$, if $f \in L^{p}(E)$ then $\mathcal{G}(f)$ is contained in $L^{p}(E)$. In this paper, we will consider rearrangements of bounded functions. If $f_{0} \in L^{\infty}(E)$, we denote by $\overline{\mathcal{G}\left(f_{0}\right)}$ the weak ${ }^{*}$ closure of $\mathcal{G}\left(f_{0}\right)$ in $L^{\infty}(E) ; \overline{\mathcal{G}\left(f_{0}\right)}$ can be characterized by the following property.

Proposition 5. Let $f_{0} \in L^{\infty}(E)$. Then $\overline{\mathcal{G}\left(f_{0}\right)}=\left\{f \in L^{\infty}(E): f \prec f_{0}\right\}$.

The claim follows from Theorem 22.13 and Theorem 22.2 in [12].

Proposition 6. (Hardy-Littlewood inequality) Let $E \subset \mathbb{R}^{N}$ be a set of finite measure and $f, g: E \rightarrow \mathbb{R}$ two measurable functions such that $|f|^{*}|g|^{*} \in L^{1}(0,|E|)$. Then $f g \in L^{1}(E)$ and

$$
\int_{E} f(x) g(x) d x \leq \int_{0}^{|E|} f^{*} g^{*} d s .
$$

Moreover, there exists $\tilde{g} \sim g$ such that, inserted in (6) in place of $g$, gives the equality sign.

The above proposition follows immediately from Theorem 10.1 and Theorem 11.1 in $[12]$.

Corollary 1. Let $E \subset \mathbb{R}^{\mathbb{N}}$ be a set of finite measure, $f: E \rightarrow \mathbb{R}$ a measurable function such that $|f|^{*} \in L^{1}(0,|E|)$. Then, $f \in L^{1}(A)$ for every measurable subset $A$ of $E$ of measure $t \in(0,|E|)$ and the identity

$$
\int_{0}^{t} f^{*} d s=\sup _{\substack{A \subseteq E \\|A|=t}} \int_{A} f d x
$$

holds.

Proof. In Proposition 6, choose $g=\chi_{A}$ for any measurable subset $A$ of $E$ of measure $t$. By (6) we find

$$
\int_{A} f d x=\int_{E} f \chi_{A} d x \leq \int_{0}^{|E|} f^{*}\left(\chi_{A}\right)^{*} d s=\int_{0}^{t} f^{*} d s .
$$

The subset $A$ being arbitrary in $E$, we can write

$$
\sup _{\substack{A \subseteq E \\|A|=t}} \int_{A} f d x \leq \int_{0}^{t} f^{*} d s .
$$

Now, fix $A$ in (8) and apply the second part of Proposition 6: there exists $\tilde{g} \sim \chi_{A}$ such that

$$
\int_{E} f \tilde{g} d x=\int_{0}^{|E|} f^{*} \tilde{g}^{*} d s
$$

By Definition 1, it is not difficult to show that $\tilde{g} \sim \chi_{A}$ implies $\tilde{g}=\chi_{\tilde{A}}$ for a suitable set $\tilde{A}$ of measure $t$. Thus, we find

$$
\int_{\tilde{A}} f d x=\int_{0}^{t} f^{*} d s
$$

which concludes the proof.

Finally, we state Theorem 18.10 in [12] for our case.

Proposition 7. Let $E \subset \mathbb{R}^{N}$ be a set of finite measure and $f_{1}, f_{2}, g \in L^{1}(E)$. If $g \prec f_{1}+f_{2}$, then there exist $g_{1}, g_{2} \in L^{1}(E)$ such that $g=g_{1}+g_{2}$ with $g_{1} \prec f_{1}$ and $g_{2} \prec f_{2}$. 
2.3. The minimization of $\boldsymbol{\lambda}_{\mathbf{1}}(\boldsymbol{m})$. As mentioned in Section 1, many authors investigated the minimization of $\lambda_{1}(m)$ as a function of the weight $m$ in connection with mathematical ecology. Such a minimization is examined over a class of functions which reflects some biological constraints.

Here we are mainly concerned with the papers [5] and [9]. In particular, in [9] the authors consider the optimization of $\lambda_{1}(m)$ as $m$ varies in a class of rearrangements. Their results about existence and characterization of a minimizer $\check{m}$ of $\lambda_{1}(m)$ can be summarized and adapted to our treatment as follows.

Proposition 8. Let $\Omega \subset \mathbb{R}^{N}$ be a bounded smooth domain and $m_{0} \in L^{\infty}(\Omega)$ such that $\left|\left\{x \in \Omega: m_{0}(x)>0\right\}\right|>0$. Then, there exists a solution $\check{m} \in \mathcal{G}\left(m_{0}\right)$ of the minimization problem

$$
\inf \left\{\lambda_{1}(m): m \in \overline{\mathcal{G}\left(m_{0}\right)}\right\} .
$$

Furthermore, if $\check{m} \in \mathcal{G}\left(m_{0}\right)$ is an arbitrary solution of (9) and $u_{\check{m}}$ denotes the unique positive eigenfunction normalized by $\left\|u_{\check{m}}\right\|_{H_{0}^{1}(\Omega)}=1$ of (1) with $m=\check{m}$ and $\lambda=$ $\lambda_{1}(\check{m})$, then there exists an increasing function $\psi$ such that $\check{m}=\psi\left(u_{\check{m}}\right)$ a.e. in $\Omega$.

Proof. By Theorem 2.1 of [9], there is a solution $\check{m} \in \mathcal{G}\left(m_{0}\right)$ of the problem $\inf \left\{\lambda_{1}(m): m \in \mathcal{G}\left(m_{0}\right)\right\}$ and, for any such minimizer, there exists an increasing function $\psi$ such that $\check{m}=\psi\left(u_{\check{m}}\right)$ a.e. in $\Omega$. By Proposition 3.6 in [1], $\overline{\mathcal{G}\left(m_{0}\right)}$ is weakly* compact and metrizable in the weak* topology. Then, since $\mathcal{G}\left(m_{0}\right)$ is dense in $\overline{\mathcal{G}\left(m_{0}\right)}$ and by Proposition 2.1 of [9], any minimizer $\check{m} \in \mathcal{G}\left(m_{0}\right)$ above solves problem (9) as well.

Remark 1. Reasoning as in the proof of Theorem 1.1 in [1], it can be shown that all the minimizers of problem $(9)$ belong to $\mathcal{G}\left(m_{0}\right)$. As already noted after Theorem 1, we prefer to include this result in a future paper.

As secondary result, in Section 4 we show how Theorem 3.9 in [5] can be regarded as a corollary of Proposition 8.

\section{Sums of closures of classes of rearrangements}

In this section we prove a formula about the classes of rearrangements of measurable functions, which we did not find in the literature. Let $E \subset \mathbb{R}^{N}$ be a set of finite measure.

Definition 5. Let $V$ be a real vector space, $A_{1}, \ldots, A_{n} \subseteq V$ and $\alpha \in \mathbb{R}$. We define

$$
\sum_{i=1}^{n} A_{i}=\left\{v \in V: v=\sum_{i=1}^{n} a_{i}, a_{i} \in A_{i}, i=1, \ldots, n\right\}
$$

and

$$
\alpha A=\{v \in V: v=\alpha a, a \in A\} .
$$

As stated in the following theorem, when $A_{i}=\overline{\mathcal{G}\left(f_{i}\right)}$ for some bounded functions $f_{i}, i=1, \ldots, n$, their sum is equal to $\overline{\mathcal{G}\left(\sum_{i=1}^{n} f_{i}\right)}$ provided every pair of functions $f_{i}, f_{j}$ realizes the equality in $(6)$.

Theorem 3. Let $f_{1}, \ldots, f_{n} \in L^{\infty}(E)$ be such that $\int_{E} f_{i} f_{j} d x=\int_{0}^{|E|} f_{i}^{*} f_{j}^{*} d s$ for all $i, j=1, \ldots, n$. Then

$$
\sum_{i=1}^{n} \overline{\mathcal{G}\left(f_{i}\right)}=\overline{\mathcal{G}\left(\sum_{i=1}^{n} f_{i}\right)}
$$


Proof. First, we show that $\overline{\mathcal{G}\left(\sum_{i=1}^{n} f_{i}\right)} \subseteq \sum_{i=1}^{n} \overline{\mathcal{G}\left(f_{i}\right)}$. Assume $h \in \overline{\mathcal{G}\left(\sum_{i=1}^{n} f_{i}\right)}$; by Proposition $5, h \prec \sum_{i=1}^{n} f_{i}$. Using Proposition $7 n-1$ times, there exists a set of $n$ integrable functions $h_{1}, \ldots, h_{n}$ such that $h=\sum_{i=1}^{n} h_{i}$ and $h_{i} \prec f_{i}$, i.e. $h_{i} \in \overline{\mathcal{G}\left(f_{i}\right)}$ for all $i=1, \ldots, n$. Therefore $h \in \sum_{i=1}^{n} \overline{\mathcal{G}\left(f_{i}\right)}$.

Second, we prove the opposite inclusion $\sum_{i=1}^{n} \overline{\mathcal{G}\left(f_{i}\right)} \subseteq \overline{\mathcal{G}\left(\sum_{i=1}^{n} f_{i}\right)}$. We claim that

$$
\left(\sum_{i=1}^{n} f_{i}\right)^{*}=\sum_{i=1}^{n} f_{i}^{*}
$$

Putting $f=\sum_{i=1}^{n} f_{i}$, using iii) of Proposition 3 (with $F$ equal to the square function), Proposition 6 and the assumption $\int_{E} f_{i} f_{j} d x=\int_{0}^{|E|} f_{i}^{*} f_{j}^{*} d s$ for $i, j=1, \ldots, n$, we have

$$
\begin{aligned}
\int_{0}^{|E|}\left(f^{*}-\sum_{i=1}^{n} f_{i}^{*}\right)^{2} d s= & \int_{0}^{|E|}\left(f^{*}\right)^{2} d s+\sum_{i=1}^{n} \int_{0}^{|E|}\left(f_{i}^{*}\right)^{2} d s \\
& -2 \sum_{i=1}^{n} \int_{0}^{|E|} f^{*} f_{i}^{*} d s+\sum_{\substack{i, j=1 \\
i \neq j}}^{n} \int_{0}^{|E|} f_{i}^{*} f_{j}^{*} d s \\
\leq & \int_{E} f^{2} d x+\sum_{i=1}^{n} \int_{E} f_{i}^{2} d x \\
& -2 \sum_{i=1}^{n} \int_{E} f f_{i} d x+\sum_{\substack{i, j=1 \\
i \neq j}}^{n} \int_{E} f_{i} f_{j} d x \\
= & \int_{E}\left(f-\sum_{i=1}^{n} f_{i}\right)^{2} d x=0
\end{aligned}
$$

which proves the claim.

Now, let $h=\sum_{i=1}^{n} h_{i} \in \sum_{i=1}^{n} \overline{\mathcal{G}\left(f_{i}\right)}$ with $h_{i} \prec f_{i}$ for $i=1, \ldots, n$. For each $t \in(0,|E|)$, using $(7),(10)$ and $h_{i} \prec f_{i}$ for $i=1, \ldots, n$, we have

$$
\begin{aligned}
\int_{0}^{t} h^{*} d s & =\int_{0}^{t}\left(\sum_{i=1}^{n} h_{i}\right)^{*} d s=\sup _{\substack{A \subseteq E \\
|A|=t}} \int_{A} \sum_{i=1}^{n} h_{i} d x \leq \sum_{i=1}^{n} \sup _{\substack{A \subseteq E \\
|A|=t}} \int_{A} h_{i} d x \\
& =\sum_{i=1}^{n} \int_{0}^{t} h_{i}^{*} d s \leq \sum_{i=1}^{n} \int_{0}^{t} f_{i}^{*} d s=\int_{0}^{t}\left(\sum_{i=i}^{n} f_{i}\right)^{*} d s ;
\end{aligned}
$$

moreover, using ii) of Proposition 3 and $h_{i} \prec f_{i}$ for $i=1, \ldots, n$, we find

$$
\begin{aligned}
\int_{0}^{|E|} h^{*} d s & =\int_{0}^{|E|}\left(\sum_{i=1}^{n} h_{i}\right)^{*} d s=\int_{E} \sum_{i=1}^{n} h_{i} d x=\sum_{i=1}^{n} \int_{0}^{|E|} h_{i}^{*} d s \\
& =\sum_{i=1}^{n} \int_{0}^{|E|} f_{i}^{*} d s=\int_{E} \sum_{i=1}^{n} f_{i} d x=\int_{0}^{|E|}\left(\sum_{i=1}^{n} f_{i}\right)^{*} d s .
\end{aligned}
$$

Therefore $h \prec \sum_{i=1}^{n} f_{i}$, i.e. $h \in \overline{\mathcal{G}\left(\sum_{i=1}^{n} f_{i}\right)}$. This completes the proof.

Even though the functions $f_{i}$ 's do not realize the equality in (6), it is still possible to write $\sum_{i=1}^{n} \overline{\mathcal{G}\left(f_{i}\right)}$ as closure of the class of rearrangements of a suitable function. This is a consequence of the following theorem. 
Theorem 4. Let $g_{1}, \ldots, g_{n} \in L^{\infty}(E)$. Then, there exist $f_{1}, \ldots, f_{n}$ such that $f_{i} \sim g_{i}$ and $\int_{E} f_{i} f_{j} d x=\int_{0}^{|E|} f_{i}^{*} f_{j}^{*} d s$ for all $i, j=1, \ldots, n$.

Proof. We put $f_{1}=g_{1}$ and by Proposition 6 we can choose $f_{2} \sim g_{2}$ such that $\int_{E} f_{1} f_{2} d x=\int_{0}^{|E|} f_{1}^{*} f_{2}^{*} d s$. As in the previous proof, we have $\left(f_{1}+f_{2}\right)^{*}=f_{1}^{*}+f_{2}^{*}$. Similarly, we can select $f_{3} \sim g_{3}$ so as to

$$
\int_{E}\left(f_{1}+f_{2}\right) f_{3} d x=\int_{0}^{|E|}\left(f_{1}+f_{2}\right)^{*} f_{3}^{*} d s
$$

or, equivalently,

$$
\int_{E} f_{1} f_{3} d x+\int_{E} f_{2} f_{3} d x=\int_{0}^{|E|} f_{1}^{*} f_{3}^{*} d s+\int_{0}^{|E|} f_{2}^{*} f_{3}^{*} d s .
$$

By the Hardy-Littlewood inequality (6) applied to the pairs $f_{1}, f_{3}$ and $f_{2}, f_{3}$, the last equation leads to

$$
\int_{E} f_{1} f_{3} d x=\int_{0}^{|E|} f_{1}^{*} f_{3}^{*} d s \text { and } \int_{E} f_{2} f_{3} d x=\int_{0}^{|E|} f_{2}^{*} f_{3}^{*} d s .
$$

In general, once we have found $f_{1}, \ldots, f_{k}$ such that $f_{i} \sim g_{i}$ and $\int_{E} f_{i} f_{j} d x=\int_{0}^{|E|} f_{i}^{*} f_{j}^{*} d s$ for all $i, j=1, \ldots, k$, we can choose $f_{k+1} \sim g_{k+1}$ such that

$$
\int_{E}\left(f_{1}+\cdots+f_{k}\right) f_{k+1} d x=\int_{0}^{|E|}\left(f_{1}+\cdots+f_{k}\right)^{*} f_{k+1}^{*} d s .
$$

Using again (6) we conclude that $\int_{E} f_{i} f_{j} d x=\int_{0}^{|E|} f_{i}^{*} f_{j}^{*} d s$ for all $i, j=1, \ldots, k+1$. After a finite number of steps we find all the functions $f_{1}, \ldots, f_{n}$ of the statement.

Remark 2. As noted above, from Theorem 3 and Theorem 4 it follows that for arbitrary bounded functions $g_{1}, \ldots, g_{n}$, there exist $f_{1}, \ldots, f_{n}$, with $f_{1} \sim g_{1}, \ldots, f_{n} \sim$ $g_{n}$, such that

$$
\sum_{i=1}^{n} \overline{\mathcal{G}\left(g_{i}\right)}=\overline{\mathcal{G}\left(\sum_{i=1}^{n} f_{i}\right)}
$$

We also note that the product of the closure of a class of rearrangements by a real number is again a closure of a class of rearrangements.

Theorem 5. Let $f$ be a bounded measurable function. Then

$$
\alpha \overline{\mathcal{G}(f)}=\overline{\mathcal{G}(\alpha f)}
$$

for all $\alpha \in \mathbb{R}$.

Proof. It is an immediate consequence of (ii) in [12, Lemma 8.2].

\section{Classes of weights $m(x)$ and proof of Theorem 1}

In this section $\Omega \subset \mathbb{R}^{N}$ will denote a measurable set of finite measure. In [5] the authors considered the class of weights

$$
\begin{aligned}
\mathcal{M}= & \left\{m(x) \in L^{\infty}(\Omega):-m_{2} \leq m(x) \leq m_{1} \text { a.e. in } \Omega, \int_{\Omega} m d x=m_{3}\right. \\
& \text { and } m(x)>0 \text { on a set of positive measure }\}
\end{aligned}
$$


where $m_{1}, m_{2}$ and $m_{3}$ are constants with $m_{1}$ and $m_{2}$ positive and $-m_{2}|\Omega|<m_{3} \leq$ $m_{1}|\Omega|$. They prove that there exists a solution of the problem

$$
\inf \left\{\lambda_{1}(m): m \in \mathcal{M}\right\}
$$

of the form $m_{1} \chi_{E}-m_{2} \chi_{\Omega \backslash E}$, where $E$ is a measurable subset of $\Omega$ such that $m_{1}|E|-$ $m_{2}|\Omega \backslash E|=m_{3}$. Therefore, problem (13) can be recast as a shape optimization problem on the set $\mathcal{N}=\left\{m_{1} \chi_{E}-m_{2} \chi_{\Omega \backslash E}: E \subseteq \Omega\right.$ is measurable, with $m_{1}|E|-$ $\left.m_{2}|\Omega \backslash E|=m_{3}\right\}$. In fact, as it is shown in the following lemmas, the set $\mathcal{N}$ coincides with a class of rearrangements and $\mathcal{M}$ can be written by means of the weak* closure of $\mathcal{N}$. Therefore, problem (13) can be seen as a particular case of problem (9).

Lemma 1. Let $\Omega \subset \mathbb{R}^{N}$ be a measurable set of finite measure and $m_{1}, m_{2}, m_{3}$ constants such that $-m_{2}|\Omega| \leq m_{3} \leq m_{1}|\Omega|$. Then, the set of functions $\mathcal{N}=\left\{m_{1} \chi_{E}-\right.$ $m_{2} \chi_{\Omega \backslash E}: E \subseteq \Omega$ is measurable, with $\left.m_{1}|E|-m_{2}|\Omega \backslash E|=m_{3}\right\}$ coincides with the class of rearrangements $\mathcal{G}\left(m_{0}\right)$, where $m_{0}$ is an arbitrary fixed element of $\mathcal{N}$.

Proof. If an equality sign holds in $-m_{2}|\Omega| \leq m_{3} \leq m_{1}|\Omega|$, then the set $\mathcal{N}$ reduces to a singleton containing a constant function. In this case the statement is trivially true, hence in the rest of the proof we assume $-m_{2}|\Omega|<m_{3}<m_{1}|\Omega|$. Clearly, for any element of $\mathcal{N}$, the set $E$ has measure $e=\left(m_{2}|\Omega|+m_{3}\right) /\left(m_{1}+m_{2}\right)$. We recall that a class of rearrangements is an equivalence class with respect to the equimeasurability relation among measurable functions. First, we show that all the elements of $\mathcal{N}$ are equimeasurable. This follows immediately from the identity

$$
|\{x \in \Omega: f(x)>t\}|= \begin{cases}|\Omega| & \text { if } t<-m_{2}, \\ e & \text { if }-m_{2} \leq t<m_{1}, \\ 0 & \text { if } t \geq m_{1}\end{cases}
$$

for each $f \in \mathcal{N}$. Now, let $f$ be a measurable function which satisfies (14). We will show that $f \in \mathcal{N}$ and this will complete the proof. For abbreviation, by $\{f>t\}$ we mean $\{x \in \Omega: f(x)>t\}$ and similarly for $\{f=t\}$ and $\{f \geq t\}$. Applying elementary measure theory to the identity $\{f \geq t\}=\cap_{k=1}^{\infty}\{f>t-1 / k\}$ for $t=m_{1},-m_{2}$ and using (14) we find $\left|\left\{f \geq m_{1}\right\}\right|=e$ and $\left|\left\{f \geq-m_{2}\right\}\right|=|\Omega|$. Finally, from $|\{f=t\}|=$ $|\{f \geq t\}|-|\{f>t\}|$ and (14) again for $t=m_{1},-m_{2}$, we get $\left|\left\{f=m_{1}\right\}\right|=e$ and $\left|\left\{f=-m_{2}\right\}\right|=|\Omega|-e$, which imply $f \in \mathcal{N}$.

In other words, $\mathcal{N}=\mathcal{G}\left(m_{0}\right)$ for any $m_{0} \in \mathcal{N}$. As the following lemma shows, it turns out that the class $\mathcal{M}$ considered in [5, Theorem 3.9] is equal to the subset of the weak* closure of $\mathcal{G}\left(m_{0}\right)$ in $L^{\infty}(\Omega)$ containing the functions which are positive in a subset of $\Omega$ of positive measure.

Lemma 2. Let $\Omega \subset \mathbb{R}^{N}$ be a measurable set of finite measure and $\mathcal{F}=\{m(x) \in$ $L^{\infty}(\Omega):-m_{2} \leq m(x) \leq m_{1}$ a.e. in $\Omega$ and $\left.\int_{\Omega} m d x=m_{3}\right\}$, with $m_{1}, m_{2}, m_{3}$ constants and $-m_{2}|\Omega| \leq m_{3} \leq m_{1}|\Omega|$. Moreover, let $e=\left(m_{2}|\Omega|+m_{3}\right) /\left(m_{1}+m_{2}\right)$ if $m_{1}+m_{2}>0$ and $e=|\Omega|$ if $m_{1}+m_{2}=0$. Then $\mathcal{F}=\overline{\mathcal{G}\left(m_{0}\right)}$, where $m_{0}$ is an arbitrary function such that $m_{0}^{*}=m_{1} \chi_{(0, e)}-m_{2} \chi_{[e,|\Omega|)}$ or, equivalently, is of the form $m_{0}=m_{1} \chi_{E_{0}}-m_{2} \chi_{\Omega \backslash E_{0}}$, where $E_{0} \subseteq \Omega$ has measure $e$.

Proof. As in Lemma 1 the case $m_{1}+m_{2}=0$ is obvious; therefore hereafter we assume $m_{1}+m_{2}>0$. Let $m \in \overline{\mathcal{G}\left(m_{0}\right)}$. By Proposition 5 and ii) of Proposition 4 , we have $-m_{2} \leq m(x) \leq m_{1}$ a.e. in $\Omega$. Moreover, by ii) of Proposition 3, Proposition 5 and Definition 3 we find 


$$
\int_{\Omega} m d x=\int_{0}^{|\Omega|} m^{*} d s=\int_{0}^{|\Omega|} m_{0}^{*} d s=\int_{\Omega} m_{0} d x=m_{3} .
$$

Therefore, $m \in \mathcal{F}$ and then $\overline{\mathcal{G}\left(m_{0}\right)} \subseteq \mathcal{F}$.

Now assume $m \in \mathcal{F}$. Using i) and ii) of Proposition 3 we obtain $-m_{2} \leq m^{*}(x) \leq$ $m_{1}$ a.e. in $\Omega$ and

$$
\int_{0}^{|\Omega|} m^{*} d s=\int_{\Omega} m d x=m_{3}=\int_{\Omega} m_{0} d x=\int_{0}^{|\Omega|} m_{0}^{*} d s .
$$

Fix $t \leq e$; we have

$$
\int_{0}^{t} m^{*} d s \leq \int_{0}^{t} m_{1} d s=\int_{0}^{t} m_{0}^{*} d s .
$$

If, instead $t \geq e$, we find

$$
\begin{aligned}
\int_{0}^{t} m^{*} d s & =\int_{0}^{|\Omega|} m^{*} d s-\int_{t}^{|\Omega|} m^{*} d s \leq \int_{0}^{|\Omega|} m^{*} d s+\int_{t}^{|\Omega|} m_{2} d s \\
& =\int_{0}^{|\Omega|} m_{0}^{*} d s-\int_{t}^{|\Omega|} m_{0}^{*} d s=\int_{0}^{t} m_{0}^{*} d s .
\end{aligned}
$$

Then, by Definition 3 and Proposition 5 we find $m \in \overline{\mathcal{G}\left(m_{0}\right)}$ and then $\mathcal{F} \subseteq \overline{\mathcal{G}\left(m_{0}\right)}$. The proof is completed.

By using Proposition 8, Lemmas 1 and 2 we obtain an alternative proof of Theorem 3.9 in [5], which we recall (with our notation) here for the convenience of the reader.

Theorem 6. [5, Theorem 3.9] Let $\Omega \subset \mathbb{R}^{N}$ be a bounded smooth domain. Let $\mathcal{M}=\left\{m(x) \in L^{\infty}(\Omega):-m_{2} \leq m(x) \leq m_{1}\right.$ a.e. $\Omega, m(x)>0$ on a set of positive measure and $\left.\int_{\Omega} m(x) d x=m_{3}\right\}$, with $m_{1}, m_{2}$ and $m_{3}$ constants such that $m_{1}$ and $m_{2}$ are positive and $-m_{2}|\Omega|<m_{3} \leq m_{1}|\Omega|$. Then, there exists a measurable set $E \subseteq \Omega$ such that $\check{m}=m_{1} \chi_{E}-m_{2} \chi_{\Omega \backslash E} \in \mathcal{M}$ and $\lambda_{1}(\check{m})=\inf \left\{\lambda_{1}(m): m \in \mathcal{M}\right\}$.

Proof. Let $L^{+}=\left\{m(x) \in L^{\infty}(\Omega):|\{m(x)>0\}|>0\right\}$. By Lemma 2 we have $\mathcal{M}=\overline{\mathcal{G}\left(m_{0}\right)} \cap L^{+}$, where $m_{0}=m_{1} \chi_{E_{0}}-m_{2} \chi_{\Omega \backslash E_{0}}$ and $E_{0}$ is an arbitrary measurable subset of $\Omega$ of measure $\left(m_{2}|\Omega|+m_{3}\right) /\left(m_{1}+m_{2}\right)$. By Proposition 8 (being $m_{0} \in L^{+}$), there exists a minimizer $\check{m} \in \mathcal{G}\left(m_{0}\right)$ of the problem (9). Note that $m_{0}$ belongs to the set $\mathcal{N}$ of Lemma 1 ; thus, by the same lemma, $\mathcal{G}\left(m_{0}\right)=\mathcal{N}$, which implies $\check{m}=m_{1} \chi_{E}-m_{2} \chi_{\Omega \backslash E}$ for some subset $E$ of $\Omega$ such that $|E|=\left(m_{2}|\Omega|+m_{3}\right) /\left(m_{1}+m_{2}\right)$. Moreover, $\check{m} \in L^{+}$and then $\check{m} \in \mathcal{M}$. Finally, $\check{m}$ being a minimizer in $\overline{\mathcal{G}\left(m_{0}\right)}$, it minimizes $\lambda_{1}(m)$ in $\mathcal{M}$ as well. This concludes the proof.

We now have all the necessary tools to prove Theorem 1.

Proof of Theorem 1. Let $L^{+}=\left\{m(x) \in L^{\infty}(\Omega):|\{m(x)>0\}|>0\right\}$. By Lemma 2 we have $\mathcal{F}_{i}=\overline{\mathcal{G}\left(f_{i}\right)}$, where $f_{i}=q_{i} \chi_{E_{i}}-p_{i} \chi_{\Omega \backslash E_{i}}$, with $E_{i} \subseteq \Omega$ has measure

$$
e_{i}=\frac{p_{i}|\Omega|+l_{i}}{p_{i}+q_{i}}, \quad i=1,2 .
$$

By Theorem 4 , we can replace $f_{1}$ and $f_{2}$ (however, we use the same symbols), without changing their class of rearrangements, in such a way that $\int_{\Omega} f_{1} f_{2} d x=\int_{0}^{|\Omega|} f_{1}^{*} f_{2}^{*} d s$; 
moreover, by Lemma $1, f_{1}$ and $f_{2}$ are still of the form written above. By Theorem 3 , we have $\overline{\mathcal{G}\left(f_{1}\right)}+\overline{\mathcal{G}\left(f_{2}\right)}=\overline{\mathcal{G}\left(f_{1}+f_{2}\right)}$; thus,

$$
\mathcal{M}=\left(\mathcal{F}_{1}+\mathcal{F}_{2}\right) \cap L^{+}=\left(\overline{\mathcal{G}\left(f_{1}\right)}+\overline{\mathcal{G}\left(f_{2}\right)}\right) \cap L^{+}=\overline{\mathcal{G}\left(f_{1}+f_{2}\right)} \cap L^{+}=\overline{\mathcal{G}\left(m_{0}\right)} \cap L^{+}
$$

where $m_{0}=f_{1}+f_{2}$. Arguing as in the proof of Theorem 3 we find $\left(f_{1}+f_{2}\right)^{*}=f_{1}^{*}+f_{2}^{*}$, which, exploiting the form of $f_{1}$ and $f_{2}$, implies

$$
\left(f_{1}+f_{2}\right)^{*}=\left(q_{1}+q_{2}\right) \chi_{(0, \gamma)}+r \chi_{[\gamma, \delta)}-\left(p_{1}+p_{2}\right) \chi_{[\delta,|\Omega|)},
$$

where $\gamma=\min \left\{e_{1}, e_{2}\right\}, \delta=\max \left\{e_{1}, e_{2}\right\}$ and

$$
r= \begin{cases}q_{1}-p_{2} & \text { if } e_{1}>e_{2} \\ 0 & \text { if } e_{1}=e_{2} \\ q_{2}-p_{1} & \text { if } e_{1}<e_{2} .\end{cases}
$$

Equality (17) implies that $m_{0}$ is a weight of the form

$$
\left(q_{1}+q_{2}\right) \chi_{G_{0}}+r \chi_{E_{0} \backslash G_{0}}-\left(p_{1}+p_{2}\right) \chi_{\Omega \backslash E_{0}}
$$

for two subsets $G_{0}, E_{0}$ of $\Omega$ such that $G_{0} \subseteq E_{0}$ and $\left|G_{0}\right|=\gamma,\left|E_{0}\right|=\delta$. By Proposition 8 (being $m_{0} \in L^{+}$), there exists a minimizer $\check{m} \in \mathcal{G}\left(m_{0}\right)$ of the problem (9). Reasoning similarly as we did in the proof of Lemma 1, we find that any weight in $\mathcal{G}\left(m_{0}\right)$ has the same form as $m_{0}$. Then we have

$$
\check{m}=\left(q_{1}+q_{2}\right) \chi_{G}+r \chi_{E \backslash G}-\left(p_{1}+p_{2}\right) \chi_{\Omega \backslash E}
$$

for two subsets $G, E$ of $\Omega$ such that $G \subseteq E$ and $|G|=\gamma,|E|=\delta$. Moreover, $\check{m} \in L^{+}$ and, by (16), $\check{m} \in \mathcal{M}$. Clearly, $\check{m}$ is a minimizer in $\mathcal{M}$.

Let $\check{f}_{i} \in \mathcal{F}_{i}, i=1,2$, such that $\check{m}=\check{f}_{1}+\check{f}_{2}$. Since the subsets $G, E \backslash G, \Omega \backslash E$ form a partition of $\Omega$ and $-p_{i} \leq \check{f}_{i} \leq q_{i}, i=1,2$, a.e. in $\Omega$, we obtain

$$
\check{f}_{1}=q_{1} \chi_{G}+\check{f}_{1} \chi_{E \backslash G}-p_{1} \chi_{\Omega \backslash E}
$$

and

$$
\check{f}_{2}=q_{2} \chi_{G}+\check{f}_{2} \chi_{E \backslash G}-p_{2} \chi_{\Omega \backslash E} .
$$

If $e_{1}=e_{2}$ we have $|E \backslash G|=\delta-\gamma=0$ and $G=E$, which yield the statement in this case. Let us now assume $e_{1} \neq e_{2}$. Integrating $\check{f}_{i}$ over $\Omega$ and because $\check{f}_{i} \in \mathcal{F}_{i}, i=1,2$, we get

$$
q_{i} \gamma+\int_{E \backslash G} \check{f}_{i} d x-p_{i}(|\Omega|-\delta)=l_{i}, \quad i=1,2,
$$

which, by using (15), gives

$$
\int_{E \backslash G} \check{f}_{i} d x=q_{i}\left(e_{i}-\gamma\right)-p_{i}\left(\delta-e_{i}\right), \quad i=1,2,
$$

and then

$$
\int_{E \backslash G} \check{f}_{i} d x=\left\{\begin{array}{ll}
q_{i}(\delta-\gamma) & \text { if } e_{i}=\delta, \\
-p_{i}(\delta-\gamma) & \text { if } e_{i}=\gamma,
\end{array} \quad i=1,2 .\right.
$$

Recalling that $|E \backslash G|=\delta-\gamma$ and $-p_{i} \leq \check{f}_{i} \leq q_{i}, i=1,2$, we conclude

$$
\check{f}_{i}=\left\{\begin{array}{ll}
q_{i} & \text { if } e_{i}=\delta, \\
-p_{i} & \text { if } e_{i}=\gamma,
\end{array} \quad i=1,2,\right.
$$

a.e. in $E \backslash G$. If $e_{1}>e_{2}$ we find

$$
\check{f}_{1}=q_{1} \chi_{G}+q_{1} \chi_{E \backslash G}-p_{1} \chi_{\Omega \backslash E}=q_{1} \chi_{E}-p_{1} \chi_{\Omega \backslash E}
$$


and

$$
\check{f}_{2}=q_{2} \chi_{G}-p_{2} \chi_{E \backslash G}-p_{2} \chi_{\Omega \backslash E}=q_{2} \chi_{G}-p_{2} \chi_{\Omega \backslash G}
$$

which gives the claim of the statement for $e_{1}>e_{2}$. The case $e_{1}<e_{2}$ can be similarly treated. This concludes the proof.

We note that, with a little effort, the previous proof can be extended to the case of a finite number of sets $\mathcal{F}_{i}, i=1, \ldots, n$.

Remark 3. Let us consider an example. Suppose we have to distribute two different resources through an environment $\Omega$. The first resource $\mathcal{F}_{1}$ affects positively the survival of the population (for example food), whereas the second $\mathcal{F}_{2}$ does it negatively (for example predators). Moreover, we have two independent sets of constraints on $\mathcal{F}_{1}$ and $\mathcal{F}_{2}$. Choosing the parameters in order to keep very simple our calculation, we write

$$
\mathcal{F}_{1}=\left\{f_{1}(x) \in L^{\infty}(\Omega): 0 \leq f_{1}(x) \leq 1 \text { a.e. in } \Omega \text { and } \int_{\Omega} f_{1} d x=\frac{2|\Omega|}{3}\right\}
$$

and

$$
\mathcal{F}_{2}=\left\{f_{2}(x) \in L^{\infty}(\Omega):-1 \leq f_{2}(x) \leq 0 \text { a.e. in } \Omega \text { and } \int_{\Omega} f_{2} d x=-\frac{|\Omega|}{2}\right\} .
$$

We find $e_{1}=2|\Omega| / 3, e_{2}=|\Omega| / 2$. Since $e_{1}>e_{2}$, then an optimal location of the two resources is given by the local growth rate $\check{m}_{1}=\chi_{E}-\chi_{\Omega \backslash G}$ where $E, G$ are suitable subsets of $\Omega$ such that $E \supset G$ and $|E|=2|\Omega| / 3,|G|=|\Omega| / 2$. Note that there is a subset of $\Omega$ (i.e. $E \backslash G$ ) which contains both resources, one at its maximum density and the other at its minimum. In general, this fact occurs if and only if $e_{1} \neq e_{2}$.

It is instructive to compare $\check{m}_{1}$ with the optimal local growth rate that one finds when the two independent sets of constraints on the resources are replaced by a single set of condition on their sum, i.e. on the local growth rate $m(x)=f_{1}(x)+f_{2}(x)$. In this case we have to find a minimizer over the class

$$
\mathcal{M}=\left\{m(x) \in L^{\infty}(\Omega):-1 \leq m(x) \leq 1 \text { a.e. in } \Omega, \int_{\Omega} m d x=\frac{|\Omega|}{6}\right\} .
$$

By using Theorem 3.9 in [5], the optimal local growth rate is given by $\check{m}_{2}=\chi_{E}-\chi_{\Omega \backslash E}$, where $E$ is a subset of $\Omega$ such that $|E|=e=7|\Omega| / 12$. Using Remark 1 , since $\check{m}_{1}$ and $\check{m}_{2}$ are not equimeasurable, it can be shown that $\lambda_{1}\left(\check{m}_{2}\right)<\lambda_{1}\left(\check{m}_{1}\right)$, i.e. $\check{m}_{2}$ is a better local growth rate than $\check{m}_{1}$.

Summarizing, $\check{m}_{1}$ and $\check{m}_{2}$ are two different trade-offs: the former gives the best arrangement of the resources when the amount of each of them is fixed, the latter yields a better chance of survival of the population but it satisfies a less stringent condition on the availability of the resources.

\section{Steiner symmetry}

Following [4], we introduce the notion of Steiner symmetrization. Let $l\left(x^{\prime}\right)=$ $\left\{x=\left(x_{1}, x^{\prime}\right) \in \mathbb{R}^{N}: x_{1} \in \mathbb{R}\right\}$ for any fixed $x^{\prime} \in \mathbb{R}^{N-1}$ and let $T$ be the hyperplane $\left\{x=\left(x_{1}, x^{\prime}\right) \in \mathbb{R}^{N}: x_{1}=0\right\}$.

Definition 6. Let $E \subset \mathbb{R}^{N}$ be a measurable set. Then the set $E^{\sharp}=\left\{x=\left(x_{1}, x^{\prime}\right)\right.$ $\left.\in \mathbb{R}^{N}: 2\left|x_{1}\right|<\left|E \cap l\left(x^{\prime}\right)\right|_{1}, x^{\prime} \in \mathbb{R}^{N-1}\right\}$ is said the Steiner symmetrization of $E$ with respect to the hyperplane $T$ and $E$ is called Steiner symmetric if $E^{\sharp}=E$. 
It can easily be shown that $|E|=\left|E^{\sharp}\right|$. In the sequel, by $\{u>c\}$ we mean the set $\{x \in E: u(x)>c\}$.

Definition 7. Let $E \subset \mathbb{R}^{N}$ be a measurable set of finite measure and $u: E \rightarrow \mathbb{R}$ a measurable function bounded from below. Then the function $u^{\sharp}: E^{\sharp} \rightarrow \mathbb{R}$, defined by $u^{\sharp}(x)=\sup \left\{c \in \mathbb{R}: x \in\{u>c\}^{\sharp}\right\}$, is said the Steiner symmetrization of $u$ with respect to the hyperplane $T$ and $u$ is called Steiner symmetric if $u^{\sharp}=u$ a.e. in $E$.

It can be proved that $\left\{x \in E^{\sharp}: u^{\sharp}(x)>t\right\}=\{x \in E: u(x)>t\}^{\sharp}$ for all $t \in \mathbb{R}$. In particular, when $E$ is Steiner symmetric, $u$ and $u^{\sharp}$ are equimeasurable.

We remind some well known properties of the Steiner symmetrization:

a) if $E \subset \mathbb{R}^{N}$ is a measurable set of finite measure, $u: E \rightarrow \mathbb{R}$ is a measurable function bounded from below and $\psi: \mathbb{R} \rightarrow \mathbb{R}$ is an increasing function, then

$$
(\psi(u))^{\sharp}=\psi\left(u^{\sharp}\right) \quad \text { a.e. in } E
$$

(see [4, Lemma 3.2]);

b) if $E \subset \mathbb{R}^{N}$ is a measurable set of finite measure, $u, v: E \rightarrow \mathbb{R}$ are two measurable functions bounded from below such that $u v \in L^{1}(E)$, then the Hardy-Littlewood inequality holds:

$$
\int_{E} u(x) v(x) d x \leq \int_{E^{\sharp}} u^{\sharp}(x) v^{\sharp}(x) d x
$$

(see [4, Lemma 3.3]);

c) if $\Omega$ is a bounded domain and $u \in H_{0}^{1}(\Omega)$ is nonnegative, then $u^{\sharp} \in H_{0}^{1}\left(\Omega^{\sharp}\right)$ and the Pòlya-Szegö inequality holds:

$$
\int_{\Omega^{\sharp}}\left|\nabla u^{\sharp}(x)\right|^{2} d x \leq \int_{\Omega}|\nabla u(x)|^{2} d x
$$

(see [8, Theorem 2.1]).

The proof of Theorem 2 relies on a deep result of Cianchi and Fusco which we specialize to our case (see [8, Theorem 2.6 and Proposition 2.3]).

Proposition 9. Let $\Omega \subset \mathbb{R}^{N}$ be a Steiner symmetric bounded domain. Let $u \in H_{0}^{1}(\Omega)$ be a nonnegative function satisfying

$$
\left|\left\{\left(x_{1}, x^{\prime}\right) \in \Omega:\left(u^{\sharp}\right)_{x_{1}}\left(x_{1}, x^{\prime}\right)=0\right\} \cap\left\{\left(x_{1}, x^{\prime}\right) \in \Omega: u^{\sharp}\left(x_{1}, x^{\prime}\right)<M\left(x^{\prime}\right)\right\}\right|=0 .
$$

where $M\left(x^{\prime}\right)=\operatorname{ess} \sup \left\{u^{\sharp}\left(x_{1}, x^{\prime}\right):\left(x_{1}, x^{\prime}\right) \in \Omega \cap l\left(x^{\prime}\right)\right\}$. If equality is attained in (20), then $u^{\sharp}=u$ a.e. in $\Omega$.

Proof of Theorem 2. Let $\check{m}$ be a minimizer of problem (3); by Proposition 8 , there exists an increasing function $\psi$ such that $\check{m}=\psi\left(u_{\check{m}}\right)$, where $u_{\check{m}}$ denotes the unique positive eigenfunction normalized by $\left\|u_{\check{m}}\right\|_{H_{0}^{1}(\Omega)}=1$ of (1) with $m=\check{m}$ and $\lambda=\lambda_{1}(\check{m})$. Therefore, by property (18), the Steiner symmetry of $\check{m}$ is an immediate consequence of the Steiner symmetry of $u_{\check{m}}$. Hence, we need to show that $u_{\check{m}}^{\sharp}=u_{\check{m}}$. By using (4) with $m=\check{m}, \lambda=\lambda_{1}(\check{m}), u=u_{\check{m}}$ and letting $\varphi \rightarrow u_{\check{m}}$ in $H_{0}^{1}(\Omega)$ we find

$$
\check{\lambda}_{1}=\lambda_{1}(\check{m})=\frac{\int_{\Omega}\left|\nabla u_{\check{m}}\right|^{2} d x}{\int_{\Omega} \check{m} u_{\check{m}}^{2} d x} .
$$

The inequalities (19), (20) and property (18) yield

$$
\int_{\Omega} \check{m} u_{\check{m}}^{2} d x \leq \int_{\Omega} \check{m} \check{m}^{\sharp}\left(u_{\check{m}}^{\sharp}\right)^{2} d x \quad \text { and } \quad \int_{\Omega}\left|\nabla u_{\check{m}}\right|^{2} d x \geq \int_{\Omega}\left|\nabla u_{\check{m}}^{\sharp}\right|^{2} d x \text {. }
$$


Consequently we deduce

$$
\check{\lambda}_{1}=\frac{\int_{\Omega}\left|\nabla u_{\check{m}}\right|^{2} d x}{\int_{\Omega} \check{m} u_{\check{m}}^{2} d x} \geq \frac{\int_{\Omega}\left|\nabla u_{\check{m}}^{\sharp}\right|^{2} d x}{\int_{\Omega} \check{m} \sharp\left(u_{\check{m}}^{\sharp}\right)^{2} d x} .
$$

Exploiting (5) and the minimality of $\check{\lambda}_{1}$ we can write

$$
\frac{1}{\check{\lambda}_{1}}=\frac{\int_{\Omega} \check{m} u_{\check{m}}^{2} d x}{\int_{\Omega}\left|\nabla u_{\check{m}}\right|^{2} d x} \leq \frac{\int_{\Omega} \check{m}^{\sharp}\left(u_{\check{m}}^{\sharp}\right)^{2} d x}{\int_{\Omega}\left|\nabla u_{\check{m}}^{\sharp}\right|^{2} d x} \leq \frac{\int_{\Omega} \check{m}^{\sharp}\left(u_{\check{m}^{\sharp}}\right)^{2} d x}{\int_{\Omega}\left|\nabla u_{\check{m}^{\sharp}}\right|^{2} d x}=\frac{1}{\lambda_{1}\left(\check{m}^{\sharp}\right)} \leq \frac{1}{\check{\lambda}_{1}} .
$$

Therefore, all the previous inequalities become equalities and yield

$$
\int_{\Omega} \check{m} u_{\check{m}}^{2} d x=\int_{\Omega} \check{m} \sharp\left(u_{\check{m}}^{\sharp}\right)^{2} d x \quad \text { and } \quad \int_{\Omega}\left|\nabla u_{\check{m}}\right|^{2} d x=\int_{\Omega}\left|\nabla u_{\check{m}}^{\sharp}\right|^{2} d x ;
$$

furthermore, by (5), $u_{\check{m}}^{\sharp}$ is an eigenfunction associated to $\lambda_{1}\left(\check{m}^{\sharp}\right)$. By the simplicity of $\lambda_{1}\left(\check{m}^{\sharp}\right), u_{\check{m}}^{\sharp}$ being positive in $\Omega$ and, by $(23),\left\|u_{\check{m}}^{\sharp}\right\|_{H_{0}^{1}(\Omega)}=\left\|u_{\check{m}}\right\|_{H_{0}^{1}(\Omega)}=1$, we conclude that $u_{\tilde{m}}^{\sharp}=u_{\check{m}^{\sharp}}$.

For simplicity of notation, we put $v=u_{\tilde{m}}^{\sharp}=u_{\check{m}^{\sharp}}$. The second identity of $(23)$ will give our result provided we show that the hypothesis (21) of Proposition 9 with $u=u_{\check{m}}$ is satisfied. The rest of the proof is devoted to this task. By $(22), \check{m}^{\sharp}$ is a minimizer of (3) and $v$ is the normalized positive eigenfunction associated to $\lambda_{1}\left(\check{m}^{\sharp}\right)=\check{\lambda}_{1}$. Moreover, by Proposition 8 , there exists an increasing function $\Psi$ such that $\check{m}^{\sharp}=\Psi(v)$. Thus $v$ satisfies the problem

$$
\begin{cases}-\Delta v=\check{\lambda}_{1} \Psi(v) v & \text { in } \Omega \\ v=0 & \text { on } \partial \Omega .\end{cases}
$$

Let $\Omega_{+}=\left\{\left(x_{1}, x^{\prime}\right) \in \Omega: x_{1}>0\right\}$ and $C_{0,+}^{\infty}\left(\Omega_{+}\right)=\left\{\varphi \in C_{0}^{\infty}\left(\Omega_{+}\right): \varphi\right.$ is nonnegative $\}$. From (24) in weak form we have

$$
\int_{\Omega_{+}} \nabla v \cdot \nabla \varphi_{x_{1}} d x=\check{\lambda}_{1} \int_{\Omega_{+}} \Psi(v) v \varphi_{x_{1}} d x \quad \forall \varphi \in C_{0,+}^{\infty}\left(\Omega_{+}\right) .
$$

Being $v \in W^{2,2}(\Omega)$, we can rewrite the previous equation as

$$
-\int_{\Omega_{+}} \nabla v_{x_{1}} \cdot \nabla \varphi d x=\check{\lambda}_{1} \int_{\Omega_{+}} \Psi(v) v \varphi_{x_{1}} d x .
$$

Adding $\check{\lambda}_{1} \int_{\Omega_{+}} \Psi(v) v_{x_{1}} \varphi d x$ to both sides and since $v \in C^{1, \beta}(\bar{\Omega})$, it becomes

$$
-\int_{\Omega_{+}} \nabla v_{x_{1}} \cdot \nabla \varphi d x+\check{\lambda}_{1} \int_{\Omega_{+}} \Psi(v) v_{x_{1}} \varphi d x=\check{\lambda}_{1} \int_{\Omega_{+}} \Psi(v)(v \varphi)_{x_{1}} d x .
$$

Let us show that $\int_{\Omega_{+}} \Psi(v)(v \varphi)_{x_{1}} d x \geq 0$. By Fubini's Theorem we get

$$
\int_{\Omega_{+}} \Psi(v)(v \varphi)_{x_{1}} d x=\int_{\mathbb{R}^{N-1}} d x^{\prime} \int_{0}^{b\left(x^{\prime}\right)} \Psi(v)(v \varphi)_{x_{1}} d x_{1},
$$

where $b\left(x^{\prime}\right)=\left|\Omega \cap l\left(x^{\prime}\right)\right|_{1} / 2$.

For any fixed $x^{\prime} \in \mathbb{R}^{N-1}$, let $\alpha=\alpha\left(x_{1}\right)$ be a primitive of $(v \varphi)_{x_{1}}$ on $\left[0, b\left(x^{\prime}\right)\right]$. Since $\alpha\left(x_{1}\right)$ is continuous and $\Psi(v)$ is decreasing with respect to $x_{1}$, the Riemann-Stieltjes 
integral $\int_{0}^{b\left(x^{\prime}\right)} \Psi(v) d \alpha\left(x_{1}\right)$ is well defined (see Theorem 7.27 and the subsequent note in [2]). Moreover, by using [2, Theorem 7.8] we have

$$
\int_{0}^{b\left(x^{\prime}\right)} \Psi(v)(v \varphi)_{x_{1}} d x_{1}=\int_{0}^{b\left(x^{\prime}\right)} \Psi(v) d \alpha\left(x_{1}\right) .
$$

By $[2$, Theorems 7.31 and 7.8$]$ there exists a point $x_{0}$ in $\left[0, b\left(x^{\prime}\right)\right]$ such that

$$
\begin{aligned}
-\int_{0}^{b\left(x^{\prime}\right)} \Psi(v) d \alpha\left(x_{1}\right) & =-\Psi\left(v\left(0, x^{\prime}\right)\right) \int_{0}^{x_{0}} d \alpha\left(x_{1}\right)-\Psi\left(v\left(b\left(x^{\prime}\right), x^{\prime}\right)\right) \int_{x_{0}}^{b\left(x^{\prime}\right)} d \alpha\left(x_{1}\right) \\
& =-\Psi\left(v\left(0, x^{\prime}\right)\right) \int_{0}^{x_{0}}(v \varphi)_{x_{1}} d x_{1}-\Psi\left(v\left(b\left(x^{\prime}\right), x^{\prime}\right)\right) \int_{x_{0}}^{b\left(x^{\prime}\right)}(v \varphi)_{x_{1}} d x_{1} .
\end{aligned}
$$

Computing the integrals and recalling that $\varphi \in C_{0,+}^{\infty}\left(\Omega_{+}\right), v$ is positive and $\Psi(v)$ is decreasing, we conclude that

$$
-\int_{0}^{b\left(x^{\prime}\right)} \Psi(v) d \alpha\left(x_{1}\right)=v\left(x_{0}, x^{\prime}\right) \varphi\left(x_{0}, x^{\prime}\right)\left[\Psi\left(v\left(b\left(x^{\prime}\right), x^{\prime}\right)\right)-\Psi\left(v\left(0, x^{\prime}\right)\right)\right] \leq 0 .
$$

Therefore, by the previous inequality and (27) it follows $\int_{0}^{b\left(x^{\prime}\right)} \Psi(v)(v \varphi)_{x_{1}} d x_{1} \geq 0$ for any $x^{\prime} \in \mathbb{R}^{N-1}$ and, in turn, from (26) we obtain $\int_{\Omega_{+}} \Psi(v)(v \varphi)_{x_{1}} d x \geq 0$. Hence, by $(25), v_{x_{1}}$ satisfies the differential inequality

$$
\Delta v_{x_{1}}+\check{\lambda}_{1} \Psi(v) v_{x_{1}} \geq 0 \quad \text { in } \Omega_{+}
$$

in weak form. Then, applying [18, Theorem 2.5.3] and since $v_{x_{1}} \leq 0$ in $\Omega_{+}$, we conclude that either $v_{x_{1}} \equiv 0$ or $v_{x_{1}}<0$. The former would lead to the contradiction $v \equiv 0$ in $\Omega_{+}$. Consequently, we have $v_{x_{1}}<0$ in $\Omega_{+}$. Similarly it can be shown that $v_{x_{1}}>0$ in $\Omega_{-}=\left\{\left(x_{1}, x^{\prime}\right) \in \Omega: x_{1}<0\right\}$. Thus

$$
\left\{\left(x_{1}, x^{\prime}\right) \in \Omega: v_{x_{1}}\left(x_{1}, x^{\prime}\right)=0\right\} \cap\left\{\left(x_{1}, x^{\prime}\right) \in \Omega: v\left(x_{1}, x^{\prime}\right)<M\left(x^{\prime}\right)\right\}=\emptyset,
$$

where $M\left(x^{\prime}\right)=\operatorname{ess} \sup \left\{v\left(x_{1}, x^{\prime}\right):\left(x_{1}, x^{\prime}\right) \in \Omega \cap l\left(x^{\prime}\right)\right\}$. Hence, by Proposition 9 with $u=u_{\check{m}}$, we find $u_{\check{m}}^{\sharp}=u_{\check{m}}$ and, finally, $\check{m}^{\sharp}=\check{m}$. This proves the theorem.

Remark 4. The counterpart of this theorem in the case of the fractional Laplacian operator has been proved in [1]. It is somewhat surprising that, in the fractional setting, the proof is much more simple.

As particular cases of Theorem 2 we obtain the following corollaries which guarantee the symmetry of the optimal sets in Theorem 1 and in Theorem 3.9 in [5].

Corollary 2. Let $\Omega \subset \mathbb{R}^{N}$ be a bounded smooth domain and assume it is Steiner symmetric with respect to the hyperplane $T=\left\{x=\left(x_{1}, x^{\prime}\right) \in \mathbb{R}^{N}: x_{1}=0\right\}$. Moreover, let $E$ and $G$ be the measurable subsets of $\Omega$ in the statement of Theorem 1 . Then, $E$ and $G$ are Steiner symmetric relative to $T$.

Proof. Applying Theorem 2 to the minimizer $\check{m}$ of $\lambda_{1}(m)$ over $\mathcal{G}\left(m_{0}\right)$ in the proof of Theorem 1, we deduce that $\check{m}$ is a Steiner symmetric function. By the claim after Definition 7, any superlevel set of $\check{m}$ is Steiner symmetric with respect to $T$; this provides the Steiner symmetry of $E$ and $G$.

In particular, when $\Omega$ is a ball, Corollary 2 specializes to the following assertion.

Corollary 3. Let $\Omega$ be a ball in $\mathbb{R}^{N}$ and let $E$ and $G$ be the measurable subsets of $\Omega$ in the statement in Theorem 1. Then, $E$ and $G$ are balls concentric with $\Omega$. 
Corollary 4. Let $\Omega \subset \mathbb{R}^{N}$ be a bounded smooth domain and assume it is Steiner symmetric with respect to the hyperplane $T=\left\{x=\left(x_{1}, x^{\prime}\right) \in \mathbb{R}^{N}: x_{1}=0\right\}$. Moreover, let $E$ be the measurable subsets of $\Omega$ in the statement of Theorem 3.9 in [5]. Then, $E$ is Steiner symmetric relative to $T$.

Proof. Applying Theorem 2 to the minimizer $\check{m}$ of $\lambda_{1}(m)$ over $\mathcal{G}\left(m_{0}\right)$ in the proof of Theorem 6 , we deduce that $\check{m}$ is a Steiner symmetric function. By the claim after Definition 7, any superlevel set of $\check{m}$ is Steiner symmetric with respect to $T$; this provides the Steiner symmetry of $E$.

Finally, when $\Omega$ is a ball we conclude with the following result.

Corollary 5. Let $\Omega$ be a ball in $\mathbb{R}^{N}$ and let $E$ be the measurable subsets of $\Omega$ in the statement in Theorem 3.9 in [5]. Then, $E$ is a ball concentric with $\Omega$.

Acknowledgments. The authors would like to thank the three anonymous referees for their very helpful comments and valuable suggestions of improvement. The authors are partially supported by the research project Evolutive and stationary Partial Differential Equations with a focus on biomathematics (Fondazione di Sardegna 2019). The authors are members of GNAMPA (Gruppo Nazionale per l'Analisi Matematica, la Probabilità e le loro Applicazioni) of INdAM (Istituto Nazionale di Alta Matematica "Francesco Severi").

\section{References}

[1] Anedda, C., F. Cuccu, and S. Frassu: Steiner symmetry in the minimization of the first eigenvalue of a fractional eigenvalue problem with indefinite weight. - Canad. J. Math. 73:4, 2021, 970-992.

[2] Apostol, T. M.: Mathematical analysis. - Addison-Wesley, 1974.

[3] Berestycki, H., F. Hamel, and L. Roques: Analysis of the periodically fragmented environment model: I - Species persistence. - J. Math. Biol. 51, 2005, 75-113.

[4] Brock, F.: Rearrangements and applications to symmetry problems in PDE. - In: Handbook of Differential Equations, Stationary Partial Differential Equations, Vol. 4, Ch. 1 (edited by M. Chipot), Elsevier BV, 2007, 1-60.

[5] Cantrell, R.S., and C. Cosner: Diffusive logistic equations with indefinite weights: population models in disrupted environments. - Proc. R. Soc. Edinb. Sect. A 112:3-4, 1989, 293-318.

[6] Cantrell, R. S., and C. Cosner: The effects of spatial heterogeneity in population dynamics. - J. Math. Biol. 29, 1991, 315-338.

[7] Chanillo, S., D. Grieser, M. Imai, K. Kurata, and I. Ohnishi: Symmetry breaking and other phenomena in the optimization of eigenvalues for composite membranes. - Commun. Math. Phys. 214:2, 2000, 315-337.

[8] Cianchi, A., and N. Fusco: Steiner symmetric extremals in Pólya-Szegö-type inequalities. Adv. Math. 203:2, 2006, 673-728.

[9] Cosner, C., F. Cuccu, and G. Porru: Optimization of the first eigenvalue of equations with indefinite weights. - Adv. Nonlinear Stud. 13:1, 2013, 79-95.

[10] Cox, S. J., and J. R. McLaughlin: Extremal eigenvalue problems for composite membranes, I. - Appl. Math. Optim. 22, 1990, 153-167.

[11] Cox, S. J., and J. R. McLaughlin: Extremal eigenvalue problems for composite membranes, II. - Appl. Math. Optim. 22, 1990, 169-187.

[12] DAY, P. W.: Rearrangements of measurable functions. - Dissertation (Ph.D.), California Institute of Technology, 1970. 
[13] De Figueiredo, D. G.: Positive solutions of semilinear elliptic problems. - In: Differential equations (edited by A. Dold and B. Eckmann), Lecture Notes in Math. 957, Springer, 1982, 34-87.

[14] Gilbarg, D., and N.S. Trudinger: Elliptic partial differential equations of second order. Springer, Berlin, Heidelberg, 1977.

[15] JhA, K., and G. Porru: Minimization of the principal eigenvalue under Neumann boundary conditions. - Numer. Funct. Anal. Optim. 32:11, 2011, 1146-1165.

[16] Lamboley, J., A. Laurain, G. Nadin, and Y. Privat: Properties of optimizers of the principal eigenvalue with indefinite weight and Robin conditions. - Calc. Var. Partial Differential Equations 55:144, 2016, 1-37.

[17] Lou, Y., and E. YAnAGIDA: Minimization of the principal eigenvalue for an elliptic boundary value problem with indefinite weight, and applications to population dynamics. - Jpn J. Indust. Appl. Math. 23:275, 2006, 275-292.

[18] PuCCI, P., and J. SERrin: Maximum principles for elliptic partial differential equations. - In: Handbook of Differential Equations: Stationary Partial Differential Equations, Vol. 4, Ch. 6 (edited by M. Chipot), Elsevier BV, 2007, 355-483.

[19] Roques, L., and F. HAMEL: Mathematical analysis of the optimal habitat configurations for species persistence. - Math. Biosci. 210:1, 2007, 34-59.

[20] Shigesada, N., and K. Kawasaki: Biological invasions: theory and practice. - Oxford Series in Ecology and Evolution, Oxford University Press, 1997.

[21] Skellam, J. G.: Random dispersal in theoretical populations. - Biometrika 38:1/2, 1951, 196-218.

Received 28 January 2021 • Accepted 21 May 2021 • Published online 13 January 2022

Claudia Anedda

University of Cagliari, Department of

Mathematics and Computer Science

Via Ospedale 72, Cagliari, 09124, Italy

canedda@unica.it
Fabrizio Cuccu

University of Cagliari, Department of

Mathematics and Computer Science

Via Ospedale 72, Cagliari, 09124, Italy

fcuccu@unica.it 